\title{
FAST MOTION IMAGING USING REDUCED FIELD OF VIEW PARTIAL FOURIER MRI
}

\author{
Harsh K. Agarwal, Khaled Z. Abd-Elmoniem, Jerry L. Prince \\ Image Analysis and Communication Laboratory, \\ Department of Electrical and Computer Engineering, \\ Johns Hopkins University, Baltimore, MD
}

\begin{abstract}
Fast MR imaging techniques often exploit the redundancy present in an underlying MR image time series to compensate for $k$-space undersampling. When imaging motion using techniques like HARP, DENSE, and phase contrast (PC), it is the phase in static regions that is constant and therefore redundant. In this paper, we present a technique that first estimates the phase in the static portion of an undersampled MR image time series and then uses a partial Fourier reconstruction technique to combine phase in the static portion and undersampled data to reconstruct the full image time series. The technique is illustrated using a computational phantom undergoing simulated cardiac motion and imaged using the HARP protocol. Results demonstrate a gradual degradation of accuracy with loss of data due to undersampling, indicating that a $25 \%$ speedup in imaging time is possible for an image time series in which $50 \%$ of the pixels correspond to the object that do not move over time.
\end{abstract}

Index Terms - MRI, Partial Fourier MR image reconstruction, rFOV, HARP.

\section{INTRODUCTION}

Fast MR imaging techniques often exploit the redundancy present in an underlying MR image time series (hereafter referred to as an image series) in order to compensate for data undersampling. Since MR imaging data is acquired in $k$ space (Fourier space), undersampling is accomplished by omitting $k$-space lines during acquisition. In such cases, data redundancy is exploited in order to compensate for the undersampled Fourier data.

One class of fast MR imaging techniques, referred to as partial Fourier MR image reconstruction (PFR) techniques [1, 2], can reduce data acquisition time by up to a factor of two. The ideal form of these techniques assumes that the underlying MR image is real-valued and use the conjugate symmetry property of real-valued signals to reconstruct the image from half of its Fourier data. In practice, however, MR images are complex-valued; for example, image phase can be caused by

\footnotetext{
This work is supported by NIH/NHLBI research grant (R01HL47405)
}

B0-field inhomogeneity and object susceptibility variations. Therefore, practical PFR techniques acquire one half of the $k$-space and additional samples of the low frequency portion of the other half of $k$-space. These methods then estimate the image phase from the low frequency samples (both halves of $k$-space), remove the phase of the whole data, and then fill in the remaining Fourier samples using conjugate symmetry. Images are reconstructed by inverse Fourier transforming the augmented data.

Reduced field of view (rFOV) techniques [3-6] represent another class of fast MR imaging techniques that make use of redundancy in the MR image series. The idea is to use the fact that in typical image series - e.g., for imaging motion or perfusion - only a part of the field of view is changing, that is the dynamic part. Over the course of an entire image series, therefore, there is redundant Fourier data representing that part of the images that does not change over time, that is the static part. Accordingly, these techniques first estimate the static portion of the image using data acquired over the whole image series. Then, Fourier data from each time instant is used together with knowledge of the static portion (which is unchanging) to estimate the dynamic portion of the image at that time instant. Data reduction is possible because the static portion is effectively imaged a bit at a time over the whole image series.

In this paper, we present a technique for fast imaging involving MR protocols that measure motion using phase-based techniques, such as HARP [7], DENSE [8] and phase contrast [9]. In these approaches, phase is used to store the displacement $[7,8]$ or velocity [9] of tissues, and it is therefore the phase that remains constant in static tissues, even though the magnitude may be changing in these regions. Our technique belongs to both PFR and $\mathrm{rFOV}$ classes of fast MR imaging techniques. As in rFOV techniques, our method first estimates the image phase in the static portion. We then use a novel PFR technique to reconstruct the MR image when the phase is known over part of the image. We demonstrate the application of our technique using a computer simulation that mimics the acquisition of MR tagging data of the heart. Results show that $25 \%$ reduction of data is possible without significant loss of accuracy when $50 \%$ of the data is static. 


\section{THEORY}

MR scanners acquire data in the Fourier transform domain or $k$-space of the underlying object, and images are reconstructed using an inverse Fourier transform operation. The two cardinal directions in $k$-space are referred to as the phase encode and frequency encode directions. A single phase encode acquisition corresponds to a line of $k$-space in the frequency encode direction, i.e., a row in $k$-space. A phase encode acquisition is normally assumed to be acquired instantaneously since it occurs fast relative to the motion of the underlying object. Fast MR imaging techniques aim to reconstruct MR images from fewer numbers of phase encode lines than would normally be required.

We describe our method in two parts. First, we present a matrix formulation that is used to reconstruct images from reduced Fourier data coupled with knowledge of the locations of both the static and dynamic portions of the images. Then, we describe an approach to estimate the phase of the static portion of the image.

\subsection{Matrix Formulation}

For the acquisition protocol described above, each row of $k$ space can be inverse Fourier transformed (one-dimensional) immediately upon acquisition because each row contains dense Fourier samples. Application of the 1-D Fourier transform to the columns cannot be immediately applied, however, because there are missing rows. For the assumed scenario, the problem we are solving is one-dimensional, since it can be applied to each column separately. In its simplest form, the static regions correspond to a collection of rows, which means the same solution applies to each column in $k$-space. This is the situation that we assume here, though it is straightforward to formulate a solution that would apply to more complicated static regions.

We aim to reconstruct $f(x)$, a column of the underlying image from its Fourier transform $F(k)$ using

$$
F(k)=\sum_{x=0}^{N-1} f(x) e^{-j 2 \pi k x / N}
$$

We rewrite the above expression in matrix form as

$$
\mathbf{F}=M \mathbf{f}
$$

where $\mathbf{F}=[F(0), F(1), \ldots, F(N-1)]^{T}$, $\mathbf{f}=[f(0), f(1), \ldots, f(N-1)]^{T}$, and $M=\left[m_{k x}=e^{-j 2 \pi k x / N}\right]$.

Let $\theta(x), x=0, \ldots, N-1$, denote an estimate of phase over the given column of an image. Since, the phase estimate (see next section) is reliable only over the static portion of the image, it follows that

$$
\operatorname{Im}\left\{f(x) e^{-j \theta(x)}\right\}=0 \quad x \in \text { static part } .
$$

Eq. (2) should be satisfied wherever we observe a Fourier sample, and Eq. (3) should be satisfied in the static part of the image. The following minimization problem captures these goals in the first two terms and provides a regularization in the third term:

$$
\min _{\mathbf{f}}\|S(\mathbf{F}-M \mathbf{f})\|^{2}+\|\Lambda \operatorname{Im}\{\Theta \mathbf{f}\}\|^{2}+\delta\|\mathbf{f}\|^{2}
$$

Here, $\Theta$ is a diagonal matrix with diagonal entries $e^{-j \theta(x)}$, $x=0, \ldots, N-1 ; S$ is a diagonal matrix with $k^{\text {th }}$ diagonal entry equals 1 if $F(k)$ is sampled, 0 otherwise; $\Lambda$ is a diagonal matrix with $x^{\text {th }}$ diagonal entry equals $\lambda$ if $x$ belongs to static portion, 0 otherwise. The solution to Eq. (4) can be found by solving the following linear system:

$$
\left[\begin{array}{c}
\operatorname{Re}\{\mathbf{F}\} \\
\operatorname{Im}\{\mathbf{F}\} \\
0 \\
0 \\
0
\end{array}\right]=\left[\begin{array}{cc}
S \operatorname{Re}\{M\} & -S \operatorname{Im}\{M\} \\
S \operatorname{Re}\{M\} & S \operatorname{Im}\{M\} \\
\Lambda \operatorname{Im}\{\Theta\} & -\Lambda \operatorname{Re}\{\Theta\} \\
\delta I & 0 \\
0 & \delta I
\end{array}\right]\left[\begin{array}{c}
\operatorname{Re}\{\mathbf{f}\} \\
\operatorname{Im}\{\mathbf{f}\}
\end{array}\right]
$$

This formulation is similar to one proposed by Bydder et al. [10]. The difference is that here $\Lambda$ is spatially varying and $\mathbf{M}$ is a matrix of DFT coefficients instead of an aliasing matrix.

\subsection{Phase Estimation}

Phase estimation plays an important role in PFR techniques since incorrect phase estimation will introduce errors and artifacts in the reconstructed images. Conventionally, PFR techniques estimate phase over an image by low pass filtering the acquired data at each time instant. This type of phase estimation accounts for only slowly varying component of phase, i.e., phase arising mainly due to B0 field inhomogeneity.

Here, we estimate phase by forming an image that is the average from the series of acquired data. Consider the series of data acquired at each location in $k$-space. We assume that our $k$-space sampling pattern yields at least one sample at each $k$-space location over the image series data acquisition process. The average $k$-space value over the entire time series is computed at each $k$-space location, forming an average Fourier transform image. An "average image" is then computed by inverse Fourier transform, and a phase estimate within the static region is extracted by the usual arctan operation.

This procedure works since averaging complex data having the same phase preserves the phase. This happens in the static region, but not in the dynamic region.

\section{SIMULATION METHODS}

We use a cardiac motion and MR tagging simulator software package to study the performance of the proposed method [11]. Cardiac motion is modeled over one heart beat to generate a tagged cardiac MR image series comprising 16 cardiac 


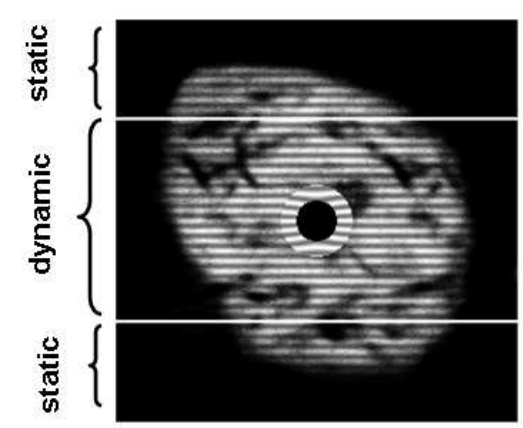

Fig. 1. Tagged image at $8^{\text {th }}$ time frame showing the static and dynamic parts of the image.

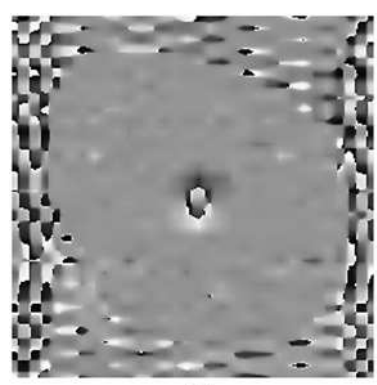

a)

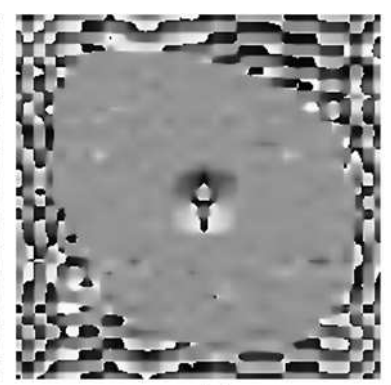

b)
Fig. 2. (a) Estimated phase from the average image when $25 \%$ of the data discarded to reconstruct an image. (b) True phase of the image at the 8th time frame.

phases. Each cardiac phase image is of size $256 \times 256$ with FOV $28 \mathrm{~cm}$ and tag spacing of $6 \mathrm{~mm}$. The HARP technique for estimating cardiac motion requires $k$-space data around only one of the spectral peaks (in each of two orientations) in $k$-space [7]. In the FastHARP protocol of [12], a $32 \times 32$ window of $k$-space around one of the spectral peak is assumed to be acquired at each time instant. We aim to further reduce this data by not acquiring few phase encode lines during temporal acquisition. Our simulation uses a bit-reversed cartesian $k$ space sampling pattern to select the phase encode lines used to generate the undersampled data (cf. [13]). The amount of data discarded while reconstructing an MR image is varied from $0 \%-37.5 \%$ in our simulation. Fig. 1 shows the $8^{\text {th }}$ time frame of the simulated tagged MR image series. We define the central one half of the rows in each image to be the dynamic portion and remaining rows to be static. We set $\lambda=10$ and $\delta=0.1$ for image reconstruction.

\section{RESULTS AND DISCUSSION}

Fig. 2(a) shows the estimated phase from the average image when $25 \%$ of the data is discarded; this should be compared to the true phase, shown in Fig. 2(b). Ignoring the background (where it is impossible to get a reliable phase estimate), it can

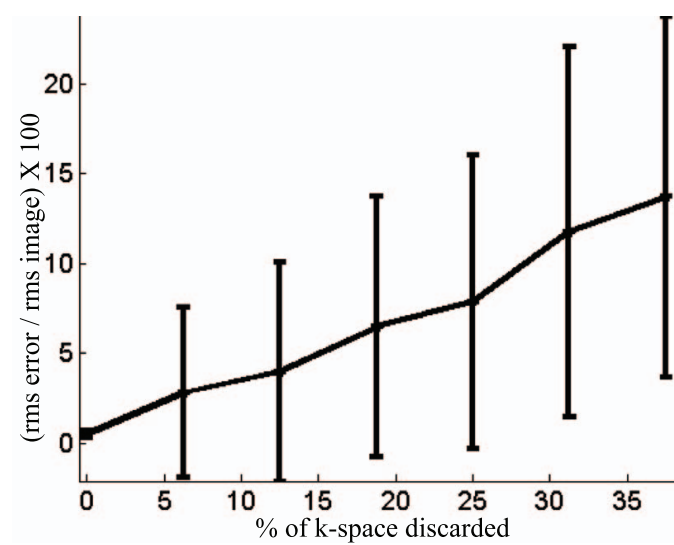

Fig. 3. Percent error in the reconstructed image plotted against the percent of $k$-space discarded. The error bars represent $+/$ - standard deviation of error over the whole time series.
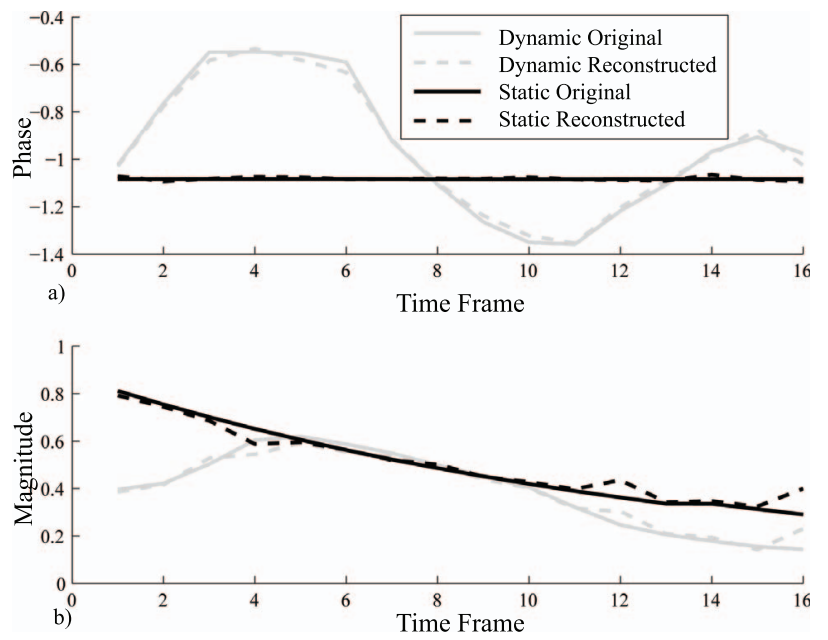

Fig. 4. Original and reconstructed a) Phase and b) Magnitude of pixel in the static and dynamic part of the image.

be observed that the phase estimate is reliable in the static portions but in error in the dynamic region (where the "heart" is). Our method is then used to reconstruct the full image series for a set of different data loss scenarios. Fig. 3 shows the percentage of image energy that is in error plotted against the percentage of data that is discarded. Note that since $50 \%$ of the data is static and phase contributes $50 \%$ of the information about a complex number, we would expect that reconstruction is possible up to a loss of $25 \%$ of the data. As shown in Figure 3, there is only $7.8 \%$ error when $25 \%$ of data is discarded. Although errors do not increase dramatically when even more than $25 \%$ of the data is discarded, visual inspection shows the presence of aliasing in the reconstructed images. Figure 4 shows the estimated and true magnitude and phase of two pixels in the image when $25 \%$ of the data is discarded. One pixel is in the "myocardium", which is moving and in the dynamic part of the image. The second pixel is in unmoving "tissue" 

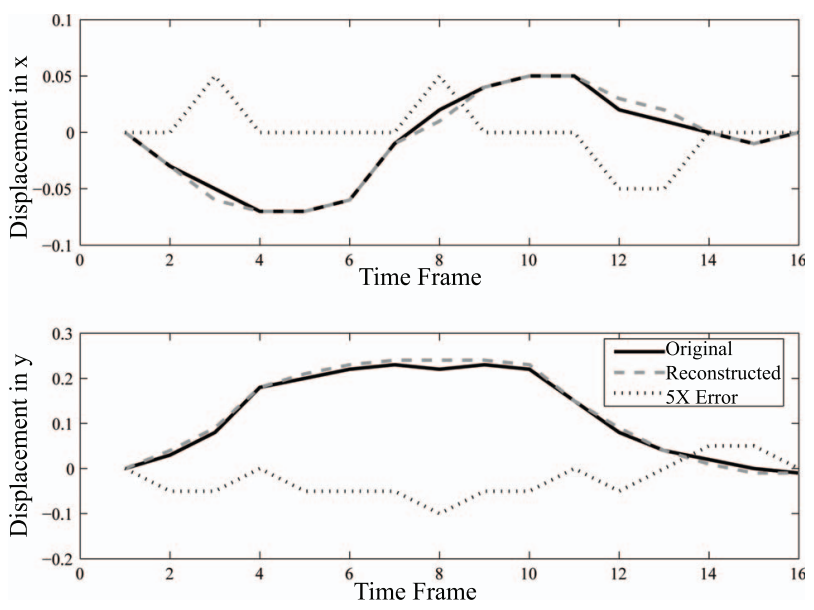

Fig. 5. $x$ and $y$ displacement of a point in the myocardium from original and reconstructed image series. A 5 times amplified error between the two displacements is also shown.

within the static part of the image. Overall, it is observed that the magnitudes and phases are reconstructed very accurately. Larger errors are observed at the $4^{\text {th }}$ and $12^{\text {th }}$ time frames are observed, however. This is because the particular $k$-space sampling pattern that we used yields a matrix [see Eq. (5)] with a larger condition number at these time frames. This error can potentially be reduced by using higher value of $\delta$ at these time instants. HARP tracking is used to compute the motion of a point in the myocardium of the heart, i.e., in the dynamic portion of the image. Fig. 5 compares the true displacements ( $x$ and $y$ ) of the point against the estimated displacements when $25 \%$ of the data is discarded. The HARP tracking algorithm is iterative, and we used $1 / 100=0.01$ pixel as a stopping criterion (this is customary). It is observed from Fig. 5 that the error is within the accuracy of our stopping criterion.

\section{DISCUSSION AND CONCLUSION}

We presented a fast MR imaging reconstruction algorithm for imaging protocols in which phase is expected to be constant over a static portion of the image time series. Our approach combines partial Fourier reconstruction concepts with reduced field of view techniques. A novel matrix inversion approach is developed to estimate a complete time series of images from partial Fourier information acquired over the time frames. A computer simulation demonstrates that $25 \%$ reduction in imaging time is possible for a $50 \%$ static part without significant loss of image reconstruction accuracy.

We demonstrated the the proposed technique for the HARP MRI protocol; it is suitable for DENSE and phase contrast as well. Our demonstration showed speedups for a bit-reversed cartesian sampling pattern; but alternate patterns, such as radial and spiral sampling trajectories, would work as well.

\section{REFERENCES}

[1] G. McGibney, M.R. Smith, S.T. Nichols, and A. Crawley, "Quantitative evaluation of several partial fourier reconstruction algorithms used in mri.," Magn. Reson. Med., vol. 30, pp. 51-59, 1993.

[2] P. Margosian, F. Schmitt, and D.E. Purdy, "Faster mr imaging: imaging with half the data.," Healthcare Instr., pp. 195-97, 1986.

[3] X. Hu and T. Parrish, "Reduction of field of view for dynamic imaging.," Magn. Reson. Med., vol. 31, pp. 691-94, 1994.

[4] K. Scheffler and J. Hennig, "Reduced circular field-ofview imaging.," Magn. Reson. Med., vol. 40, pp. 474480, 1998.

[5] J.O. Fredrickson and N.J. Pelc, "Temporal resolution improvement in dynamic imaging.," Magn. Reson. Med., vol. 35, pp. 621-625, 1996.

[6] M.E. Bummer, D. Moratal-Perez, C.Y. Hong, J. MilletRoig R.I. Pettigrew, and W.T. Dixon, "Noquist: Reduced field-of-view imaging by direct fourier inversion.," Magn. Res. Med., vol. 51, pp. 331-342, 2004.

[7] N.F. Osman and J.L. Prince, "Visualizing myocardial function using harp mri.," Phy. Med. Bio., vol. 45, no. 6, pp. 1665-1682, June 2000.

[8] A.H. Aletras, S. Ding, R.S. Balaban, and H. Wen, "Dense: Displacement encoding with stimulated echoes in cardiac functional mri.," J. Magn. Reson., vol. 137, no. 1, pp. 247-252, March 1999.

[9] N.J. Pelc, R.J. Herfkens, A. Shimakawa, and D.R. Enzmann, "Phase contrast cine magnetic resonance imaging.," Magn Reson $Q$, vol. 7, no. 4, pp. 229-54, Oct. 1991.

[10] M. Bydder and M.D. Robson, "Partial fourier partially parallel imaging.," Magn. Reson. Med.,, vol. 53, no. 6, pp. 1393-1401, May 2005.

[11] E. Waks, J.L. Prince, and A. Douglas, "Cardiac motion simulator for tagged mri.," Proc. Math. Meth. in Biomed. Image Anal., pp. 183-91, 1996.

[12] S. Sampath, J.A. Derbyshire, E. Atalar, N.F. Osman, and J.L. Prince, "Real-time imaging of two-dimensional cardiac strain using a harmonic phase magnetic resonance imaging (harp-mri) pulse sequence.," Magn. Reson. Med., vol. 50, no. 1, pp. 154-163, July 2003.

[13] H.K. Agarwal and J.L. Prince, "Theoretical framework for analyzing mr imaging of dynamic objects using filters and downsamplers.," Proc. of SPIE, vol. 6144, pp. 61441B, March 2006. 